\title{
Modeling and Identification of the Hydraulic Servo Drive
}

\author{
Piotr Woś ${ }^{1, *}$, Ryszard Dindorf ${ }^{2}$ \\ ${ }^{1}$ Faculty of Mechatronics and Machine Design, Kielce University of Technology, Kielce, 25-314, Poland \\ ${ }^{2}$ Faculty of Mechatronics and Machine Design, Kielce University of Technology, Kielce, 25-314, Poland
}

\begin{abstract}
This paper discusses possibilities to apply the dynamic identification with a discrete linear model while assessing the state of the electro-hydraulic drive dynamics. This evaluation is crucial while designing modern power or positional control systems. Experimental data is applied in order to determine the model dynamics of the real system and estimate unknown parameters of an object model. The dependencies were interpreted. The paper includes the selected results of current identification tests of the electro-hydraulic drive system as a result of which the discrete parametric object model was derived.
\end{abstract}

\section{Introduction}

Conventional control systems designed for specific working conditions operate well provided that they are subject to small deviations from these conditions. In fact, controlled objects do not fulfill the stationary conditions as a result of this interference [1]. Therefore, a problem of incomplete information about the object should not be taken into account. Incomplete information about the object, as far as hydraulic systems are concerned, usually refers to the lack of knowledge of the exact values of all parameters and signals which are applied to determine regulatory quality indicators or other optimization conditions. In the object control structures with incomplete information, algorithms providing the identification of system indeterminateness and change of the controllers' settings might be distinguished. It makes it possible to adjust the control system to variable conditions. Despite considerable advancement in on-line design of the controllers' algorithms, the industry is dominated by periodically-adjusted classic PID controllers. It is generally believed that those controllers are reliable and there are many methods of adjusting them [2]. However, due to the stability of the closed system, the methodology of adjusting the controller in real time is more modern, efficient and reliable for multidimensional control systems of high order. The standardization of those algorithms requires additional identification methods for continuous systems to be developed which might cooperate with on-line adjustable controllers. Thus, it is essential to build appropriate algorithms of parametric identification as well as appropriate algorithms of state observation. Identification is an approach to build a model of dynamic system which consists in analyzing the dependences between its input and output signals without intruding into the laws of physics governing these dependencies. The identification is applied when the rules governing the model phenomenon are either unknown or too complicated to be used for designing the model construction for control purposes. In such a situation in order to determine the model dynamics of the real system and estimate unknown parameters of an object model, experimental data is applied. The identification procedure in an iterative procedure and encompasses the following stages: planning measurements in order to obtain data, selecting a model structure in terms of its form and row of equations, estimating model parameters and verifying the model correctness by using other measurement data than used for estimating the parameters [3]. Moreover, the identification method should not be sensitive to the nature of input signals. Obtaining data sufficient for identification is only possible through a proper stimulation of system inputs, and such an interaction is not always possible in practice [4]. One method of control systems is adaptive control with model identification [5]. The model identification adaptive control (MIAC) system illustrated in Fig. 1. Identification of model is determined on the input $u$ and output $y$. Depending on these model optimal controller parameters are calculated and adjusted.

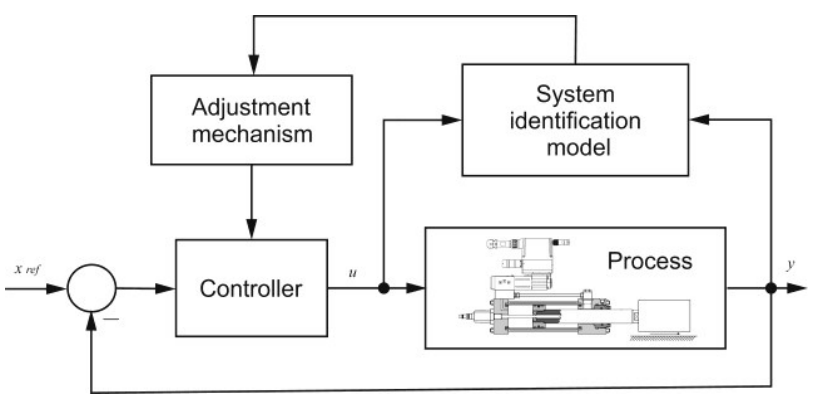

Fig. 1. Model Identification Adaptive Control (MIAC)

\footnotetext{
* Corresponding author: wos@tu.kielce.pl
} 


\section{Mathematical identification model}

The parametrized model defines precisely the bahaviour of the control object under specific conditions which are described by inputs and outputs at present and in the past. The algorithms for estimating the model coefficients were developed with the aim to track the changes of model coefficients. A typical parametric description is to present the properties of the control system in the form of discrete transmittance. The dynamic properties of the control process depend on the parameters' values of the discrete transmittance. In the synthesis of the control algorithms, the choice upon the modelling method depends to a great extent on how the suggested model is interpreted physically and whether it is easy to identify it [6]. Nonparametric models in the form of previously stored samples of the object responses, models formulated in the space of states as well as parametric models in the form of input-output discrete transmittance might be distinguished among many known descriptions [7]. Determining a parametric model describing the studied object consists in defining the model class and selecting a particular model there from. Defining the model class is limited to determining the mathematical expression in the parametric form describing the studied object. The model is selected from the accepted class by determining the numerical values of its coefficients. As a rule, foreknowledge makes it possible to establish only the model class. However, the coefficient values of the mathematical model are determined as a result of the implementation of the selected identification algorithm which stipulates how the measurement results should be processed in order to determine the values of the model coefficients. Due to the use of measurement data which is of "discrete-intime" nature, the mathematical identification models are discrete and linear in relation of the desired parameters. They take it also into account that the measurement data might be subject to mistakes as a result of introducing random interference into the models. As far as discrete time is concerned, the operator of signal backward shift by $k$ of samples $q^{-k x(n)}=x(n-k)$ is introduced to shorten the record, which gives the equation that is similar to the equations with complex argument $z^{-k}$ in the field of transforms $Z$.

In order to identify the dynamic model of the electrohydraulic servo-drive, a deterministic model with the following structure was applied:

$$
A\left(q^{-1}\right) y_{t}=B\left(q^{-1}\right) u_{t-d}+\xi_{t}
$$

where: $y_{t}, u_{t}$ - input and control signals, respectively, $d$ - discrete delay, $\xi_{t}$ - object interference.

Model (1) does not require a priori information about the identification and thus, it is relatively easy and shows no sensitivity to frequently occurring industrial changes of the structure of the control object. The disadvantage of this model is a relatively great number of previously stored samples which, however, can be used to describe objects with complex dynamics. The transmittance model in the discrete form (2) has unknown parameter values of the control object which must be identified:

$$
G(z)=\frac{B\left(z^{-1}\right)}{A\left(z^{-1}\right)}=\frac{b_{1} z^{-1}+b_{2} z^{-2}+\ldots+b_{n} z^{-n}}{1+a_{1} z^{-1}+a_{2} z^{-2}+\ldots+a_{n} z^{-n}} z^{-d}
$$

where: $a_{1}, a_{2}, \ldots, a_{n}, b_{1}, b_{2}, \ldots, b_{n}$ - parameters of the control object, $n$ - model order

\section{Algorithm of the model identification}

The process of identification is a cognitive process focused on quantitative and qualitative learning about the reality. The basis of this process is experimental research which has limited accuracy influenced by many factors, i.a.: the accuracy of the direct measurement of the object variables, influence of external disturbing factors, choice upon the signal stimulating the object with relation to its ability to identify, properties of the identification algorithm and the class of the adopted model. Both the identification process and the measurement process consist in obtaining the information about the studied object. The basis for determining the coefficients of the identification model is the measurement results of the input and output signals conducted directly on the identified object. An on-line identification algorithm was applied to provide a parametric identification of the electro-hydraulic drive. A solution for the parameters' estimation task with the use of calculation algorithms in accordance with the block diagram shown in Fig. 2 was suggested herein. The identification process consists of a few stages, the most crucial of which is the stage of numerical calculations of estimator $\hat{\theta}$.

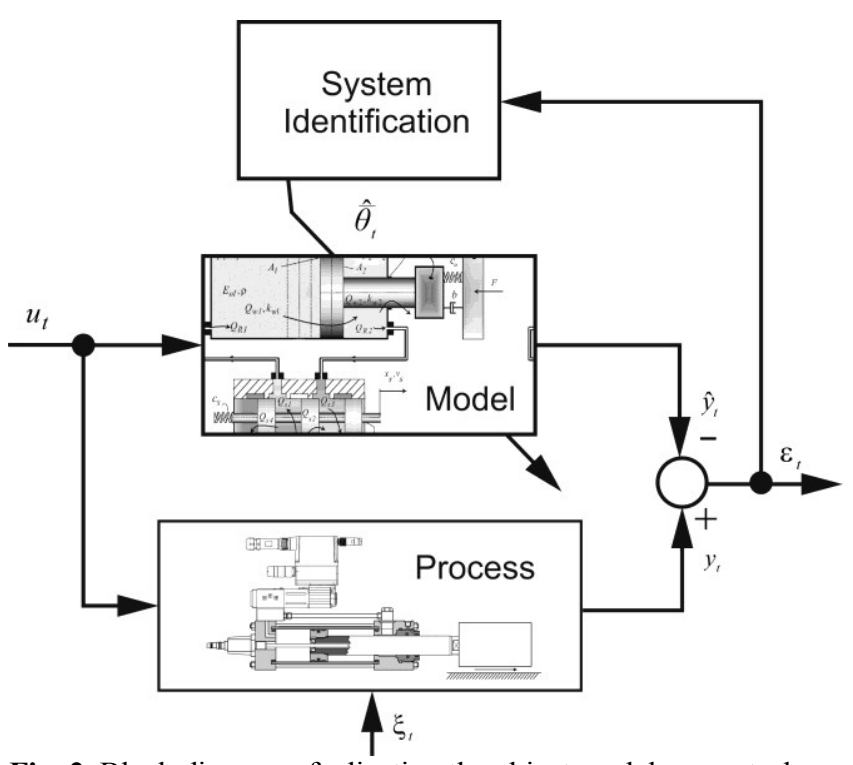

Fig. 2. Block diagram of adjusting the object model: $u_{t}$ control signal, $y_{t}$ object output signal, $\hat{y}_{t}$ output signal of the object model, $\hat{\theta}$ estimate vector of the object model parameters, $\varepsilon_{t}$ model error, $\xi$, object interference. 


\subsection{Systems with parameters variable over time}

Determining the system parameters using the method which takes the whole history of the observation process into account is the average estimation obtained throughout the complete registration period. The applied iterative algorithm does not often follow the changes in the parameters of the objects loaded with random interferences. Moreover, those systems are excessively overloaded as by increasing the number of measurements, the size of matrix $\mathbf{P}_{\mathbf{t}}$ and vector $\bar{\varphi}_{t}$ increase as well at the same time and as a consequence, the demand for the memory of the control system grows. Adjusting the iterative algorithm to tracking the object parameters variable over time leads to the method with exponential forgetfulness. The method based on RLS recursive algorithm with WRLS (Weighted Recursive Least Squares) exponential forgetfulness consists in introducing $\lambda_{t}$ forgetting coefficient, which decides upon the size of the algorithm memory, into the control equations. If the changes in parameters' values are tracked in an on-line way, estimates $\hat{\theta}$ should be determined on the basis of on-line measurements of input and output variables. Past data has no significant impact on the current object state and should be forgotten. In order to make matrix $\mathbf{P}_{t}$ independent from the past data, weight parameter $\lambda_{t}$, often called exponential forgetting coefficient, was introduced. Exponential forgetting coefficient $\lambda_{t}$ decreases the influence of the past data on the current evaluation determined with covariance matrix $\mathbf{P}_{\mathbf{t}}$ :

$$
\mathbf{P}_{t}=\left(\mathbf{P}_{\mathbf{t}-1}-\frac{\mathbf{P}_{\mathbf{t}-1} \bar{\varphi}_{t} \bar{\varphi}_{t}^{T} \mathbf{P}_{\mathbf{t}-1}}{1+\bar{\varphi}_{t}^{T} \mathbf{P}_{\mathbf{t}-1} \bar{\varphi}_{t}}\right) \frac{1}{\lambda_{t}},
$$

Then, the vector of estimated parameters takes the following form:

$$
\begin{aligned}
& \hat{\hat{\theta}_{t}}=\hat{\bar{\theta}}_{t-1}+\frac{\mathbf{P}_{t-1} \bar{\varphi}_{t}}{\lambda_{t}+\bar{\varphi}_{t}^{T} \mathbf{P}_{\mathrm{t}-1} \bar{\varphi}_{t}} \varepsilon_{t}, \\
& \varepsilon_{t}=y_{t}-\bar{\varphi}_{t}^{T} \hat{\bar{\theta}}_{t-1},
\end{aligned}
$$

Weight coefficient $\lambda_{t}$ variable over time is applied in order to accelerate the convergence of the algorithm and is determined with equation: $\lambda_{t}=\lambda^{0} \lambda_{t-1}+\left(1-\lambda_{0}\right)$, where $\lambda_{t}$ aims exponentially to 1 , while the number of measurement samples increases.

Assuming that $\lambda^{0}=\lambda_{0}=1$, the weight coefficient takes the following value: $\lambda=\lambda_{t}=1$. This value is applied while tracking low-variable parameters of the control system. For $\lambda=1$, matrix $\mathbf{P}_{t}$ depends in the case of every moment $t$ on all previous data. Due to the fact that while adjusting, the parameters were prone to changes, there is no linear dependency between vector elements $\bar{\theta}_{t}$. Introduction of this parameter results in an exponential limitation of the error signal, which means that older samples of the estimation signal error are taken to total error measurement $\varepsilon_{t}$ with correspondingly lower weight. It should be expected that lower values $\lambda<1$ will upgrade the model more quickly which is beneficial for a quicker adaptation, and simultaneously lower values $\lambda$ lead to greater temporary variations of the values of the estimated coefficients which is not desirable. That is why, the selection of forgetting coefficient $\lambda$ should be a well thought over compromise. The value of the forgetting coefficient is selected in accordance with the rate of changes of the identified object parameters as well as the level of interference included in the measurement data.
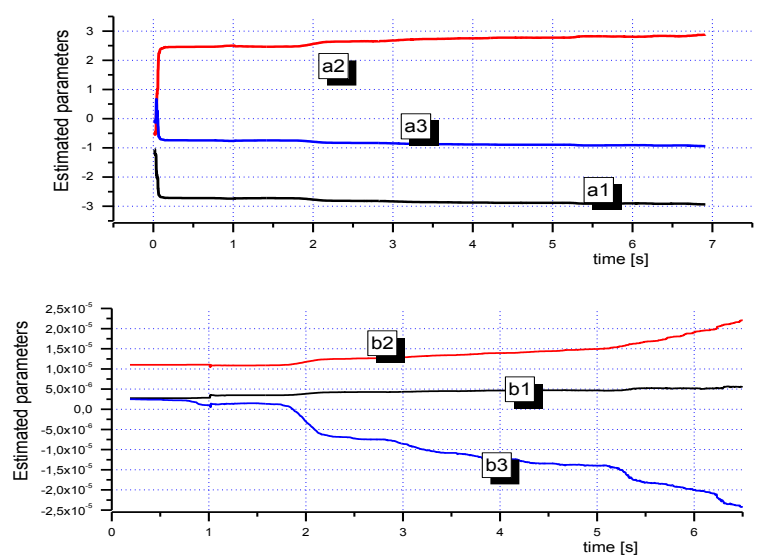

Fig. 3. Time courses of the model parameters of the control object determined using WRLS method

\subsection{Robust estimation}

A major challenge for engineers is to build such a model identification algorithm of the control object which will make it possible to accelerate the adaptation of model parameters when changing the reference signal values or the object properties. By estimating the model parameters in real time (on-line), a dynamic change of those parameters at the moment of random interference occurrence $\xi_{t}$ is possible. A solution for parameters' estimation task with the use of calculation algorithms based on RLS adaptive recursive algorithm with forgetting exponential AWRLS (Adaptive Weighted Recursive Least Squares) [8] was suggested herein. This method consists in constant $\lambda_{t}$ value adaptation during the identification process. Adopting $\lambda=$ const , as demonstrated by the attempts conducted, is difficult to be implemented in practice. In order to allow tracking the model parameters with quickly variable values, an attempt to improve RLS was made by using the adaptation of exponential forgetting coefficient $\lambda_{t}$ so that it would include the change of input and output parameters during the object identification. In this case, exponential coefficient $\lambda_{t}$ is updated at any time $t$ and recorded in the following form: 


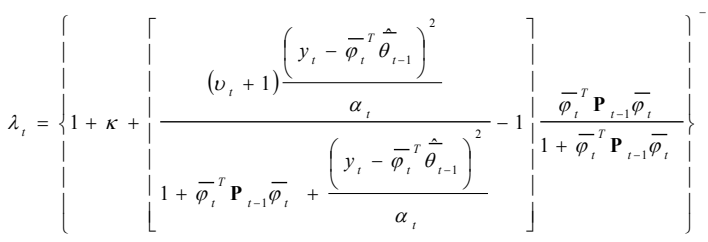

where:

$$
\begin{aligned}
& \kappa=1+\ln \left(1+\bar{\varphi}_{t}^{T} \mathbf{P}_{t-1} \bar{\varphi}_{t}\right), \\
& \alpha_{t}=\lambda_{t-1}\left|\alpha_{t-1}+\frac{\left(y_{t}-\bar{\varphi}_{t}{ }^{T} \hat{\bar{\theta}}_{t-1}\right.}{1+\bar{\varphi}_{t}{ }^{T} \mathbf{P}_{t-1} \bar{\varphi}_{t}}\right|, \\
& v_{t}=\lambda_{t-1}\left(v_{t-1}+1\right)
\end{aligned}
$$

where: $y_{t}-$ values of output size samples, $\overline{\bar{\varphi}}_{t}^{T} \hat{\overline{\theta_{t}}}-$ anticipated process output, $\mathbf{P}_{t}-$ covariance matrix.

Parameter $\lambda_{t}$ which is found in equation (6) is an adaptive weight parameter, which has a significant influence on the convergence rate of the control servodrive algorithm. If coefficient $\lambda_{t}$ aims to unity, the estimation variance decreases and the process dynamic properties deteriorate by prolonging the memory of previous measurement results. At the same time, in the states determined for a low value of coefficient $\lambda_{t}$, the estimation quality deteriorates as the variance of the generated estimation increases. Constant value tracking of the forgetting coefficient provides great process dynamics (low value of coefficient $\lambda_{t}$ ) and low random variation of the generated estimation in the event of weak stimulation (the value of coefficient $\lambda_{t}$ is close to unity, see Fig. 5). Figure 4 shows time courses of parameters $a_{i}, b_{i}$ for $n=3$ of the model order of the control object (1), determined using AWRLS while controlling the displacement of the hydraulic actuator for sinusoidal extortion.
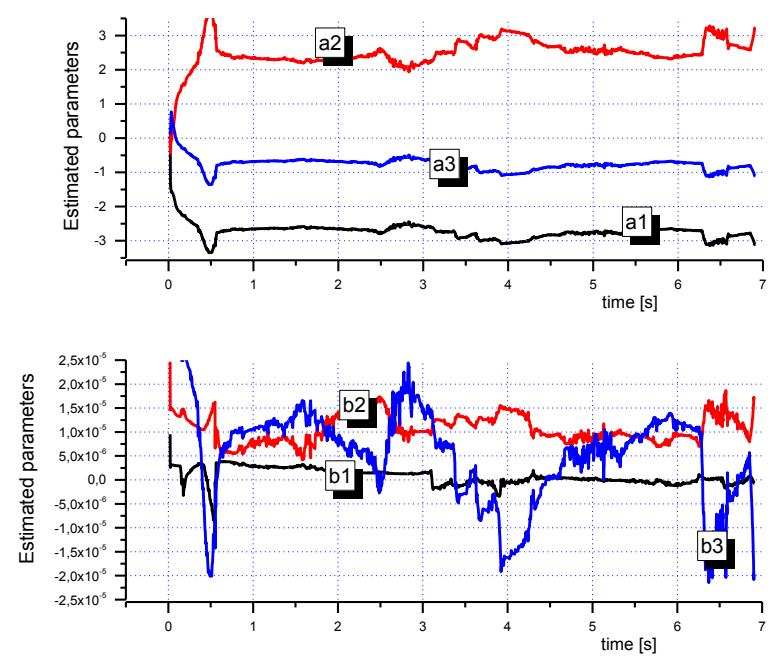

Fig. 4. Courses of identified model parameters (1) $a_{i}, b_{i}$

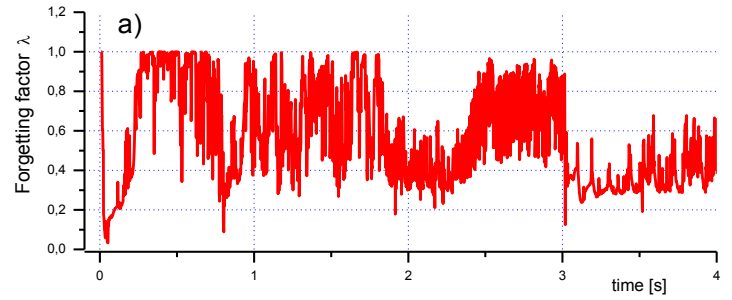

Fig. 5. Change of exponential forgetting factors $\lambda_{t}$

\section{Experimental test stand}

In the algorithms research of control electro-hydraulic servo drive is used the test stand. In the Fig. 6 presented a view of the test stand consisting of the electro-hydraulic servomechanism controlled by the proportional flow valve (2). The force is achieved by weights installed on the linear guide support (5). Servomechanism control systems is using the card of C/A converters. Displacement of the linear guide block is measured relatively to the corps test stand with the converter position Novotechnik Company (4). Velocity of the linear guide block is calculated by the differentiation piston position of the actuator (1). In the system there is also possible measurement of the technological resistance force using the force sensor (3). Hydraulic power supply is also included in the test stand $\left(P_{\max }=31.5 \mathrm{MPa}\right)$, along with the proportioned pressure valve (DBETR-10/315G24K4M-381). The test stand also includes computer system in the superior control system equipped with the Matlab/Simulink (xPC Target) software. The microcomputer has cards of converters $\mathrm{C} / \mathrm{A}$ and $\mathrm{A} / \mathrm{C}$ of the PCI type - DAS1602/16 Measurement Computing Corporation. Card along with the converter of position and force creates the measurement system [9].
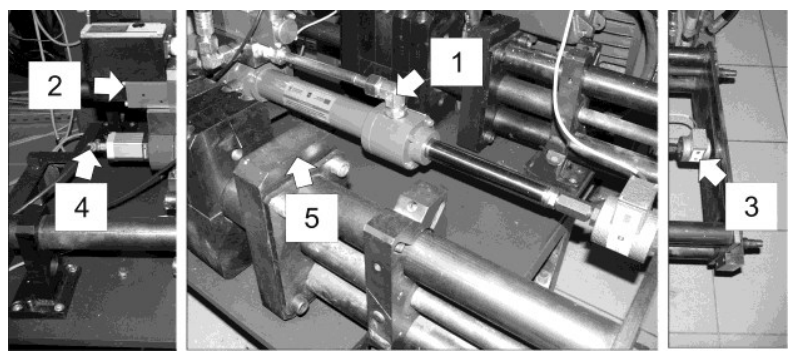

Fig. 6. View of experimental test-stand: 1 - hydraulic cylinder, 2 proportional control valve, 3 - force transducer, 4 - position transducer, 5 - slider with the mass load

\section{Model verification}

During the research, an analysis of the time course of remainders $\varepsilon$ of adjusting the model to the intended object response was conducted. Due to the random nature of remainders $\varepsilon$, statistical tests were applied which determine the autocorrelation function of remainders $R_{x}(\tau)$. The autocorrelation function (own correlation) of signal $x(t)$ determines the dependency of the values in two various moments $t$ at the distance of $\tau$ from each other. The function definition is stipulated with equation (7): 


$$
R_{x}(\tau)=\lim _{T \rightarrow \infty} \frac{1}{T} \int_{0}^{T} x(t) x(t+\tau) d \tau
$$

where: $\tau$-displacement over time.

If the model is matched in a proper way, the properties of remainders $\varepsilon$ should be characterized by symmetric distribution and be independent from input signals. They should have the properties of white noise, i.e. a stationary stochastic signal with an average value equaling to zero. This signal does not carry any information about the object reaction to the forced signal. The complete information about the object behaviour should be reproduced form the model and the process output. Figure 7 and 8 shows the courses of absolute values $|\varepsilon|$ and autocorrelation function of remainders $R_{x}(\tau)$ for model (1), type $n=2$ and $n=3$.
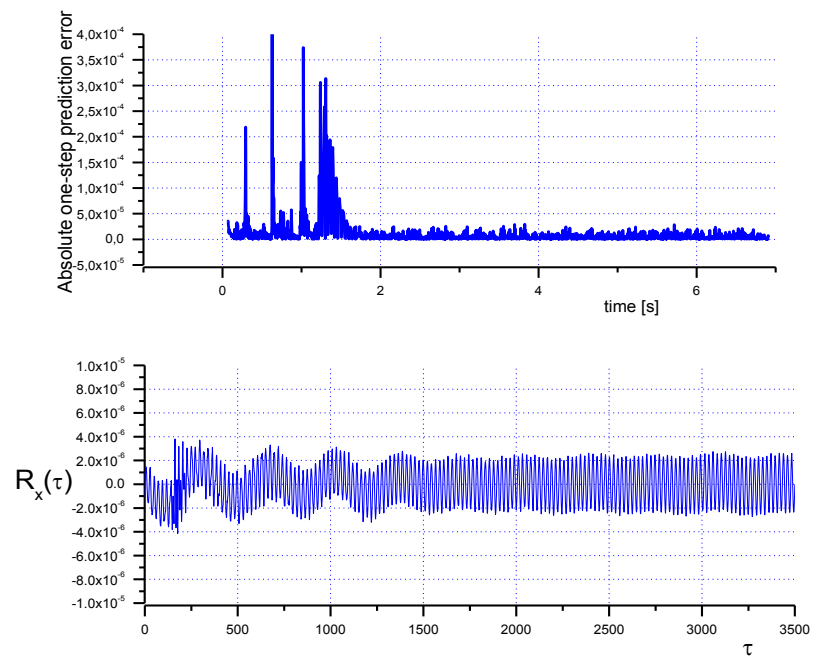

Fig. 7. The courses of absolute error $|\varepsilon|$ and autocorrelation function of remainders $R_{x}(\tau)$ for $n=2$ model
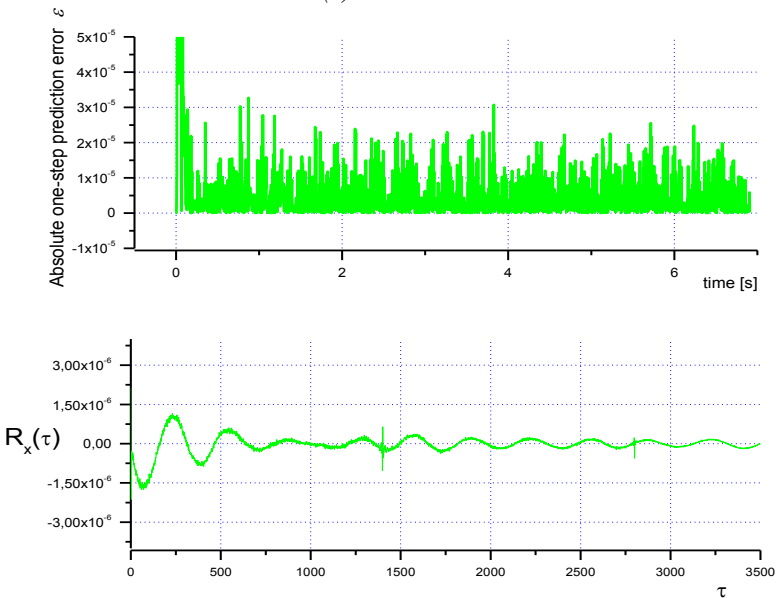

Fig. 8. The courses of absolute error $|\varepsilon|$ and autocorrelation function of remainders $R_{x}(\tau)$ for $\mathrm{n}=3$ model

If the model accuracy is measured with the autocorrelation function of remainders $R_{x}(\tau)$, then, if properly identified, it will asymptotically equal to zero for each displacement which is different than zero. From the courses presented above (see Fig. 7 and Fig. 8) it might be concluded that the best match was obtained for model $n=3$. No proper match with model $n=2$ was observed. The remainders depend on the input size, and autocorrelation function $R_{x}(\tau)$ does not aim asymptotically to zero.

\section{Summary}

An important issue regarding the selection of the adaptive controller parameters is to identify the model of the control object in a proper way. Both the model parameters and the controller parameters are selected in real time (on-line). The proper identification of the model parameters determines the quality of the electrohydraulic servo-drive control. A proper selection of appropriate tools to verify the model match for the intended object response is crucial.

\section{References}

1. B. Yao, IEEE/ASME Tran. on Mech., vol. 5, pp. 79-91, (2002).

2. M. Ahmadnezhad, M. Soltanpour, M.M.E Vol. 9, No.7, (2015).

3. M. Jelali, A. Kroll, Hydraulic Servo Systems - Modelling, Identification \& Control, Springer, (2003).

4. P. Wos, R. Dindorf, A.J. of C. No. 15, pp. 1065 $\div 1080$, (2013).

5. K. Takahashi, M. Inoue, S. Ikeo, Proc. Int. Conf. Fluid Power Trans. and Control, pp. 68-87 (1985).

6. T. Kheowree, S. Kuntanapreeda, A.J. of Con., Vol. 17, pp. 855-867, (2015).

7. M. F. Rahmat, Md. Rozali, Amer J of App. Scie. No.7, pp. 1100-1108, (2010).

8. V. Bobál, J. Böhm., J. Fessl, J. Machácek, Digital selftuning controllers : Agorithms, Implementation and Applications, Tech.\& Eng., (2005).

9. P. Wos, R. Dindorf, A.J. of C. No. 15, pp. 1065 $\div 1080$, (2013). 